\title{
Betroffene vor den Folgen kursierender sexualisierter Foto- und Videoaufnahmen schützen: Magdalena
}

„Ein halbes Jahr später und die Bilder gehen wieder in [Stadt] rum. Jeder, JEDER hat mich, also nicht richtig nackt gesehen, sondern oberkörperfrei gesehen. " (Gewaltbetroffene, Interview).

- Zusammenfassung Betroffene können von ihrer Gewaltgeschichte wiedereingeholt werden, indem sexualisierte Foto- und Videoaufnahmen oder gespeicherte Kommunikationsverläufe (z. B. archivierte Chats oder E-Mails) über digitale Medien veröffentlicht und verbreitet werden. In der Folge werden sie oftmals innerhalb ihres sozialen Umfelds gedemütigt, beschuldigt oder ausgegrenzt. All diese Folgen stellen eine Form weiterer Gewalt dar, die in einem unmittelbaren Zusammenhang mit der einst erlebten sexualisierten Gewalt steht. Die Gewaltgeschichten sind somit miteinander verwoben. Betroffene Kinder und Jugendliche haben das Gefühl, einer ausweglosen Situation ohnmächtig gegenüberzustehen. Am Beispiel einer veranschaulichenden Fallvignette setzt sich das nachfolgende Kapitel vornehmlich mit fachlichen Fragen und Abwägungsprozessen auseinander, die in Zusammenhang mit dem Schutz Gewaltbetroffener aufkommen. 


\section{Fallvignette Magdalena}

Der folgende Gesprächsauszug ist dem Protokoll des Erstgesprächs (Fachberatung gegen sexualisierte Gewalt in Kindheit und Jugend) mit der 17-jährigen Adressatin Magdalena entnommen.

Magdalena: „Es macht alles keinen Sinn mehr. Ich fühle mich hilflos. Ich war so vierzehn, ja, meine Cousine Anna war neun. Da war ich mit Anton, meinem Trainer zusammen. Das fing über Facebook an, haben viel gechattet. Persönlich war er ziemlich kalt, damit niemand was merkt, weil wir das ja nicht gedurft hätten. Aber dann kam raus, dass er mit Anna geflirtet hat. Die war auch in meinem Team. Ich habe Schluss gemacht. Trotzdem wollte er immer wieder Nacktbilder. Er hat gesagt, dass er meine Bilder rumschickt, wenn ich keine neuen schicke. Anna und ich sollten uns irgendwann zusammen fotografieren. Wir haben erst ,Nein gesagt, aber wir wollten ja nicht auffliegen. Anna wollte alles ihren Eltern erzählen, ich habe sie überredet, es zu lassen. Sie ist dann aus dem Verein ausgetreten und hat niemandem was erzählt. Anton war trotzdem sauer und hat unsere Bilder rumgeschickt. Es ging ein richtig krasser Shitstorm los. Ich war wütend auf Anton, weil er mich dafür bestraft hat, dass Anna keine Bilder mehr schickt. Es war mir zu viel, ich bin nicht mehr ins Training. Als das Angebot für das Sportinternat kam, dachte ich, das ist meine Rettung. Ich bin jetzt seit zwei Jahren hier und habe alles $800 \mathrm{~km}$ hinter mir gelassen. Bis vor ein paar Tagen. Irgendjemand hat die Nacktbilder rumgeschickt und das Gerücht verbreitet, dass ich eine Kinderfickerin bin. Alle glauben es und beleidigen mich. Niemand glaubt mir, obwohl ich es abstreite. Wenn ich gehe, habe ich nichts mehr. Wie soll ich neu anfangen, wenn mich die Gerüchte überall hin verfolgen? Die Wahrheit kann ich auch nicht sagen. Wie soll jemand verstehen, was ich Anna angetan habe?“

Sonstiges: Das Internat ist insoweit informiert, als das Kollegium Kenntnis von den im Klassenchat stattfindenden Diskreditierungen infolge der Verbreitung von Nacktfotos Magdalenas hat. Der Ursprung der Aufnahmen ist ihnen ebenso wenig bekannt wie der sexuelle Missbrauch durch Anton. Das Internat 
bemühte sich um eine gemeinsame Aufarbeitung des „Mobbings“ innerhalb der Klasse.

\section{Reflexionsfragen}

- Was löst Magdalenas Gewalterleben in uns aus?

- Wie stehen wir zu den Beleidigungen durch Magdalenas Mitschüler*innen?

- Was braucht Magdalena?

- Welche Herausforderungen sehen wir bei der Unterstützung Magdalenas?

- Welche Herausforderungen sehen wir bei der Unterstützung des Internats?

\subsection{Reflexionen zur fachlichen Einordnung des Fallbeispiels}

Magdalena sieht sich augenblicklich in einer ausweglosen Situation. Infolge kursierender Nacktfotos, die sie gemeinsam mit ihrer jüngeren Cousine Anna zeigen, werde sie von ihren Mitschüler*innen als „Kinderfickerin“ beschimpft. Sie habe diese Beschuldigung von sich zu weisen versucht, doch niemand glaube ihr. Während Magdalena in ihrer Verzweiflung der Situation entfliehen möchte, sieht sie das Risiko, dass sie die „Gerüchte überallhin verfolgen“. Erschwerend erlebt sie trotz Bemühungen des Internats nur unzureichend Schutz. Gemäß der Fallvignette wurde das „Mobbing“ aufzuarbeiten versucht. Nach Definition der Autor*innen (s. Kap. 2) handelt es sich bei der Verbreitung von Nacktfotos sowie sexistischsexualisierter Diskreditierung jedoch um mediatisierte sexualisierte Gewalt. In puncto Aufarbeitung können die Prinzipien im Umgang mit Mobbing von den Prinzipien des Umgangs mit sexualisierter Gewalt abweichen (Vobbe, 2014). In Magdalenas Ohnmachtserleben kumulieren sich also sämtliche Belastungen, die sie seit dem Wechsel in das Sportinternat zu bewältigen versucht. Vor etwa drei Jahren durchlebte sie schon einmal einen ,Shitstorm“ innerhalb ihrer Peergruppe. In ihrem damaligen Sportverein brachte ihr früherer Trainer Anton die erwähnten Nacktfotos in Umlauf. Weder damals noch heute wusste jemand, dass er diese unter Ausübung von Druck einforderte und deren Verbreitung androhte, wenn sie sich nicht gemeinsam mit ihrer Cousine Anna nackt fotografieren würde. Magdalena scheint sich für die sexualisierte Gewalt gegen Anna verantwortlich zu fühlen und befürchtet, dass niemand ihre Beweggründe nachvollziehen könne. 


\title{
8.1.1 Der Einsatz digitaler Medien verstetigt das Gewalterleben
}

Die Fallvignette ist insoweit charakteristisch für mediatisierte sexualisierte Gewalt, als existierende Foto- und Videoaufnahmen oder gespeicherte Kommunikationsverläufe (z. B. archivierte Chats oder E-Mails) oftmals dazu führen, dass Betroffene von ihrem Gewalterleben wiedereingeholt werden und erneut Gewalt erfahren.

\begin{abstract}
„Und das ist ja genau [...] das Perfide, dass das wirklich quasi zu Tathandlungen sich entwickelt, die nicht aufhören. Sozusagen selbst wenn der Täter physisch weg ist, diese Bilder zu einer Verstetigung einfach quasi der Tat werden.“
\end{abstract}

Rechtswissenschaftlerin, FGI

Die sich in Antons Besitz befindenden sexualisierten Fotos (Gewaltgeschichte 1) schaffen eine Drohkulisse, die es ermöglicht, Magdalena sexuell zu missbrauchen und sie dazu zu drängen, ihre Cousine Anna zu verwickeln (Gewaltgeschichte 2). Gleichzeitig wird mit der sanktionierenden Verbreitung (Gewaltgeschichte 3) der "Shitstorm“ im Sportverein angestoßen (Gewaltgeschichte 4) und das Ohnmachtserleben Magdalenas bestärkt. Mit der erneuten Verbreitung (Gewaltgeschichte 5) und Diskreditierung im Internat (Gewaltgeschichte 6) sieht sie sich in einer ausweglosen Situation gefangen, denn auch die Wahrheit könne sie nicht sagen: „Wie soll jemand verstehen, was ich Anna angetan habe?" Sie fürchtet, auch zukünftig von ihrer Gewaltgeschichte wiedereingeholt zu werden (Gewaltgeschichte 7). Schließlich entzog es sich bereits in der Vergangenheit ihren Einflussmöglichkeiten, eine Verbreitung zu verhindern. Die Gewaltgeschichten sind durch die kursierenden Nacktfotos medial miteinander verkettet. ${ }^{1}$ Veranschaulichend sei eine heute 15-jährige Interviewpartnerin des HUMAN-Projekts zitiert, die im Alter von 13 Jahren von ihrem damaligen Freund dazu aufgefordert wurde, ihm sexualisierte Fotos zu schicken. Seitdem kursieren die Aufnahmen wiederholt:

„Dann fing das alles in der Schule an, wo mich die Leute darauf angesprochen haben [...] und immer heftiger von überall. Und dann habe ich irgendwann dieselben Bilder auch geschickt bekommen und die sind immer wieder gekommen, immer wieder. Und immer mehr Leute. Ein halbes Jahr später und die Bilder gehen wieder in [Stadt A] rum, die sind bis nach [Stadt B], [Stadt $\mathrm{C}$ ] sind die rumgegangen. Und die ganzen

\footnotetext{
${ }^{1}$ Eine mediale Verkettung von Gewaltgeschichten bezeichnet die Bedingtheit bzw. Verwobenheit aufeinanderfolgender Gewalterfahrungen, die durch eine Mediatisierung der Gewalt - hier in Form der Existenz von Nacktfotos - ermöglicht und angestoßen wird.
} 
kannten mich einfach alle gar nicht. Jeder, JEDER hat mich, also nicht richtig nackt gesehen, sondern oberkörperfrei gesehen."

Gewaltbetroffene, Interview

Der Kreis der Empfänger*innen von Foto- oder Videoaufnahmen oder aber Gerüchten kann unüberschaubar groß werden. Die diesbezügliche Verunsicherung erleben Gewaltbetroffene meist als bedrohlich.

\subsubsection{Digitale Gewaltzeugnisse werden täter*innenstrategisch instrumentalisiert}

Digitale Gewaltzeugnisse wie sexualisierte Foto- oder Videoaufnahmen, Screenshots sexualisierter Chatverläufe oder Missbrauchsabbildungen erfüllen aus einer täter*innenstrategischen Perspektive zudem den Zweck, deren Entstehungskontext zu verkehren. Anton ist es augenscheinlich gelungen, die sexualisierte Gewalt als solche insoweit falsch darzustellen, als Magdalena diejenige ist, die von ihren Mitschüler*innen als Gewaltausübende („Kinderfickerin“) und nicht als Gewaltbetroffene gesehen wird. Dieser Prozess der Falschdarstellung basiert dabei auf einer Kontrolle a) jener Informationen, die Dritte über den Entstehungskontext der Nacktfotos erhalten sowie b) über den originären Adressat*innenkreis entsprechender Information(en). Gewaltausübende beanspruchen damit potenziell sowohl Definitionsmacht ${ }^{2}$ über Gewaltbetroffene als auch über den Gewaltkontext.

An solcherlei Formen mediatisierter sexualisierter (Peer-)Gewalt sind in aller Regel Dritte beteiligt, die als kollektiv handelnde Gruppe (Wilde, 2020) durch Vorbilder motiviert sind (Merten, 1999). Die Rolle der Vereinsmitglieder und Mitschüler*innen Magdalenas ist spätestens im Kontext der Aufarbeitung zu berücksichtigen. Nach Dekker et al. (2016) tragen nämlich ,letztlich alle gleichermaßen Verantwortung für die Konsequenzen für den Betroffenen oder die Betroffene" (ebd., S. 49). Gewaltdynamiken dieserart sind in Peerkontexten infolge kursierender Foto- oder Videoaufnahmen häufig zu beobachten (Döring, 2012). Ursächlich hierfür ist sozialpsychologischen Theorien folgend das Bedürfnis nach Anerkennung und Zugehörigkeit (Baumeister, 2013). Innerhalb des

\footnotetext{
${ }^{2}$ Unter Definitionsmacht über die eigene Person sei das Recht auf und die Möglichkeit zur Selbstbestimmung verstanden. Üben Dritte im Kontext mediatisierter sexualisierter Gewalt Definitionsmacht über die eigene Person aus, wird das Recht auf und die Möglichkeit zur selbstbestimmten Selbstdarstellung und (sexuellen) Identifikation verletzt.
} 
Kollektivs übernehmen nach Salmivalli et al. (1996) die einzelnen Gruppenmitglieder die Rollen von Täter*innen, Außenstehenden, Assistierenden oder Verstärker*innen. Täter*innen wird als Initiator*innen die führende Rolle zuteil. Damit definieren sie die geltenden Gruppennormen. An die Einzelnen wird hierbei unausgesprochen die Forderung nach Normkonformität gerichtet (König, 2016). Schäfer und Korn (2004) stellen in diesem Zusammenhang fest, dass die Assistierenden nicht nur hinsichtlich des Fortführens der Demütigung eine maßgebende Rolle einnehmen, sondern meist auch Freund*innen des/der Initiierenden sind. Ihr Handeln begründen sie häufig mit der Sorge, infolge illoyalen Verhaltens selbst als Opfer auserkoren zu werden. Abschließend sei darauf verwiesen, dass innerhalb der Peergruppe kursierende Fotos und Videos nicht notwendigerweise unter Ausübung von Gewalt entstehen. Es kann sich ebenso um Aufnahmen handeln, die konsensuell in einer vertrauensvollen Beziehung ausgetauscht wurden und anschließend nicht konsensuell weitergegeben wurden (Böhm et al., 2018; Kärgel \& Vobbe, 2020).

\subsubsection{Kursierende Gewaltzeugnisse belasten}

Das Gewalterleben hinterlässt sichtlich Spuren bei Magdalena.

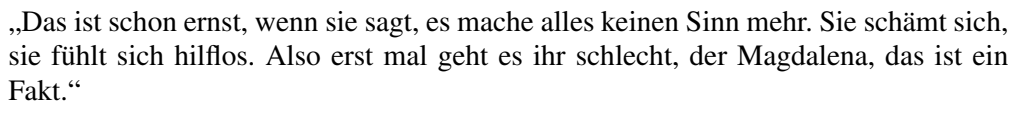

Rechtspsychologe, Gutachter, FGI

Die Sozialarbeitswissenschaft beobachtet neben kurzfristigen Belastungsmomenten aufseiten Gewaltbetroffener eine prinzipielle Sensibilisierung für „Macht-, Ohnmacht- und Abhängigkeitskonstellationen“" (Schulze \& Zimmermann, 2012, S. 28), besondere Hürden in der Bewältigung des alltäglichen Lebens, sowie das Erleben einer Tabuisierung und Stigmatisierung von Betroffenheit oder aber das Gefühl, Dritte mit der eigenen Gewaltgeschichte zu beschämen oder zu belasten (Doll \& Nagel, 2019). Die Medizin und die Klinische Psychologie diagnostizieren häufig gesundheitliche Auswirkungen, die von Symptomen depressiver Störungen über Angst- und posttraumatische Belastungsstörungen bis zur Suizidalität reichen (z. B. Cooper et al., 2016; Keupp et al., 2019). Der Verdacht einer Traumatisierung Magdalenas ist demnach nicht unbegründet. Aus qualitativen Studien zu Gewalterfahrungen im Lebensverlauf ist zudem bekannt, dass 
sich Gewalterfahrungen und Traumata aus unterschiedlichen Lebensphasen summieren. Dabei können jüngere Gewalterfahrungen Erinnerungen an vergangene verletzende oder traumatisierende Erfahrungen wecken und einstige Belastungen oder Traumafolgen reaktivieren (Kaiser, 2020; Schattenburg, 2011).

Magdalena ist höchstwahrscheinlich mehrfachbelastet. Schließlich ist davon auszugehen, dass frühere Belastungen reaktiviert werden. In diesem Zusammenhang sind die Scham- und Schuldgefühle aufgrund der Verwicklung Annas womöglich am schwerwiegendsten. So sagt sie selbst: „Die Wahrheit kann ich auch nicht sagen. Wie soll jemand verstehen, was ich Anna angetan habe?“ Eventuell schreibt sich Magdalena auch eine prinzipielle Verantwortung für die ihr widerfahrene Gewalt zu und schämt sich hierfür (vgl. Paul, 2016). Psychoanalytischen Erkenntnissen zufolge schädigen Schuldgefühle soziale Beziehungen nachhaltig, etwa aufgrund sozialen Rückzugs der von Schuld Geplagten (Kattermann, 2019; Slepian et al., 2020). Eine Medienpädagogin verweist in den Focus Group Interviews des HUMAN-Projekts hierbei auf die mögliche Verstärkung der Schuld durch die Täter*innen-Opfer-Umkehr. Darüber hinaus reaktivieren die jüngsten Belastungen (Gewaltgeschichten 5 bis 7) unter Umständen vergangene Belastungen (Gewaltgeschichten 1 bis 4), wodurch sich Magdalenas Belastungserleben potenziert. Angesichts des mehrfachen Kontrollverlusts erscheint ihr die Hoffnung auf einen Schutzraum aussichtslos. Wenn sie das Sportinternat verlasse, habe sie nichts mehr. Diese Perspektivlosigkeit hängt wahrscheinlich mit ihrer Sorge vor einer Aufdeckung zusammen. Denn als Hemmschwelle führt sie an, dass niemand verstehen würde, was sie Anna angetan habe. So fürchtet sie sich vermutlich nicht minder vor sozialer Ausgrenzung. In den Falldokumentationen des HUMAN-Projekts werden derlei Ängste als alltagsbegleitend und fortwährend beschrieben. Hierbei ist das Trigger-Potenzial ${ }^{3}$ digitaler Medien bedeutsam. Über digitale Medien ist eine Begegnung mit bzw. eine Kontaktaufnahme durch Gewaltausübende zu jeder Zeit und an jedem Ort vor, während und nach der Gewalt möglich. Zwar findet sich in der Fallvignette kein Anhaltspunkt für einen bestehenden Kontakt zu Anton, doch wird in den Falldokumentationen vergleichsweise häufig von einem fortbestehenden Täter*innen-Kontakt oder aber zufälligen Begegnungen in sozialen Netzwerken berichtet.

Aber auch etwaige Folgeerscheinungen der Diskreditierung dürfen nicht vernachlässigt werden. Der jüngeren Mobbing-Forschung zufolge kann die psychische Gesundheit wie auch das subjektive Wohlbefinden betroffener Kinder

\footnotetext{
3 Trigger sind plötzliche Erinnerungen an ein verletzendes und/oder traumatisierendes Ereignis. Reize aus der Umwelt wie z. B. Sinneseindrücke oder Erinnerungen können dies auslösen. Häufig werden dabei die damaligen Gefühle nochmals durchlebt (Jones et al., 2020).
} 
und Jugendlicher kurz- wie langfristig beeinträchtigt werden (Rosen et al., 2017; Smokowski \& Evans, 2019). Der wahrgenommene Unglaube könnte das Belastungsempfinden dahingehend verstärken (Bange, 2015). Inwieweit Magdalena darüber hinaus den Verlust der Beziehung zu Anton respektive das von ihm missbrauchte Vertrauen als belastend empfindet, ist auf Basis der Fallvignette nicht eindeutig bestimmbar. In der Gesprächsnotiz gibt sie zumindest an, wütend auf Anton zu sein, da er sie mit der Verbreitung der Nacktfotos für Annas Fehler bestrafte.

\subsection{Handlungsempfehlungen}

In Zusammenhang mit der adäquaten Fallbearbeitung sind folgende Aspekte bedeutsam:

a) Magdalena ist mehrfachbetroffen.

b) Die Gewaltwiderfahrnisse sind medial verkettet.

c) Es bestehen das Risiko sowie die Angst vor einer erneuten Verbreitung der Nacktfotos.

d) Zugleich bestehen das Risiko sowie die Angst vor einer unkontrollierbaren Aufdeckung.

e) Diese Angst verstärkt sich durch ihre Schuldgefühle.

f) Im Internat erfährt Magdalena mutmaßlich unzureichenden Schutz.

g) Es besteht infolgedessen das Risiko, dass die Geschehnisse auch bis in eine fernere Zukunft hineinwirken und eine erneute Viktimisierung begünstigen können.

Bei der adäquaten Fallbearbeitung zeichneten sich im Verlauf der Focus Group Interviews zuvorderst Kontroversen und Herausforderungen hinsichtlich des Schutzes Magdalenas ab. Verstehen wir Schutz nach Wolff (2018) als biopsychosoziales Grundbedürfnis, muss Magdalena vornehmlich bei der Abwehr aller (potenziellen) Gefahren und Gefährdungen unterstützt werden, die sie schädigen könnten. Daher sprechen wir im Folgenden Handlungsempfehlungen für den fachlichen Umgang mit den Schutzbedarfen unter Berücksichtigung des sich transzendierenden Gewaltkontexts aus. Zum Umgang mit Ängsten (Kap. 9), Belastungen durch erlebte Vertrauens- oder Beziehungsabbrüche (Kap. 4) oder aber dem Trigger-Potenzial digitaler Medien (Kap. 6 und 9) werden an anderer Stelle Empfehlungen erörtert. 
Das Gefühl von Schutz erwächst mitunter aus der subjektiven wie kognitivaffektiven Bewertung des eigenen Lebens. In diese Bewertung spielen Familienund Freundschaftsverhältnisse, materiell-ökonomische Ressourcen, Gesundheit, Emotionen, Selbstwirksamkeit, Selbstverwirklichung, verspürte Bedürfnisse kurzum die Lebenswelt - hinein (Ryff \& Keyes, 1995; Diener, 2013). Dieser Subjektivität kann im Prozess der Hilfe und Unterstützung Rechnung getragen werden, indem Magdalena von Beginn an als Expertin für ihre Lebenswelt (vgl. Kraus, 2006) adressiert wird:

- Was möchte Magdalena, von wem, ab wann, warum jetzt, wozu, mit wem, gegen wen?

- Was möchte Magdalena nicht, von wem nicht, wann noch nicht, wozu nicht?

- Mit welchen Motivationen und Erwartungen sucht Magdalena Unterstützung?

- Was soll in der Beratung geschehen, was auf keinen Fall?

Beispielhafte Fragen zur Auftragsklärung könnten etwa wie folgt lauten:

- Was müsste in dieser Sitzung geschehen, damit du am Ende sagst: ,Das hat sich gelohnt", oder ,Das hat es nicht gebracht"?

- Was versprichst du dir von der Beratung?

- Welche Einwände oder Bedenken hast du? (vgl. Haselmann, 2007, S. 174)

Anliegen und Bedürfnisse können sich jederzeit verändern, weshalb sich ein regelmäßiger Austausch empfiehlt. Grundsätzlich muss bedacht werden, dass die Erzählungen Magdalenas eventuell auf eine Kindeswohlgefährdung ${ }^{4}$ Annas hinweisen. In diesem Fall würden der gesellschaftliche Auftrag der Sozialen Arbeit (sog. Sekundärmandat) sowie deren ethische Prinzipien (sog. Tertiärmandant) Interventionen zum Schutze Annas notwendig machen, selbst wenn diese im Widerspruch zu Magdalenas Wünschen und Bedürfnissen stünden (StaubBernasconi, 2018). Umso wichtiger ist es unter diesen Umständen, Magdalena unmittelbar in sämtliche Schritte einzubeziehen.

\footnotetext{
${ }^{4}$ Näheres zur Kindeswohlgefährdung im Kontext mediatisierter sexualisierter Gewalt ist in Kap. 10 nachzulesen.
} 


\subsubsection{Bedürfnisse gemeinsam herausarbeiten und priorisieren}

Angesichts der Mehrfachbelastung sowie der medialen Verkettung der Gewaltgeschichten ist es in einem ersten Schritt geboten, die Belastungen und Bedürfnisse Magdalenas herauszuarbeiten und zu priorisieren. Die Bewältigung einer Gewaltgeschichte ist in ihren Folgen erstens nicht von der Bewältigung der anderen Gewaltgeschichten loszulösen. So darf etwa eine institutionelle Aufarbeitung der gegenwärtigen Diskreditierung nicht gegen den Willen Magdalenas zu einer Aufdeckung des originären sexuellen Missbrauchs führen. Ebenso muss bedacht werden, dass etwa das Stellen einer Strafanzeige auch Konsequenzen für Anna nach sich zieht. Zweitens sprechen Erkenntnisse der Traumatherapie für eine phasengeleitete Bewältigung, die mit Stabilisierung beginnt und mit langfristiger Traumabewältigung endet (Ehring, 2013).

\subsubsection{Systemische Feststellung von Belastungen}

Um mit Magdalena in einen Austausch über ihre wahrgenommenen Belastungen zu kommen, bieten sich nach Haselmann (2007) Frageformate an, die es erlauben, ebendiese zu skalieren, zu priorisieren und in ihrer Entstehung zu begreifen:

- Wie geht es dir momentan?

- Wie fühlst du dich, wenn du an die Verbreitung/die Beleidigung/Anna/... denkst?

- Wenn du deine augenblickliche Belastung/Traurigkeit/Schuld/Angst/... auf einer Skala von 1 bis 10 einstufen müsstest, welche Zahl würdest du angeben?

- Was bedrückt/beängstigt/... dich momentan am meisten?

- Wann hat deine Traurigkeit/Scham/Angst/... begonnen?

- Woran merkst du, dass du belastet/traurig/verängstigt/... bist?

\subsubsection{Priorisieren qua Bedürfnispyramide}

Eine Orientierung am Konzept der Bedürfnishierarchie nach Maslow (1943) ${ }^{5}$ ermöglicht es, die identifizierten Schutzbedarfe zu priorisieren. Wenngleich dieser

\footnotetext{
${ }^{5}$ Mit der Maslowschen Bedürfnishierarchie unternahm der US-amerikanische Psychologe Maslow im Jahre 1943 den Versuch, die Bedürfnisse und Motivationen des Menschen zu beschreiben und zu erklären. Er differenziert dabei zwischen physiologischen Bedürfnissen sowie Bedürfnissen nach Schutz, sozialer Verbundenheit, Anerkennung und Selbstverwirklichung. Wobei er diese insofern relational hierarchisiert versteht, als einige Bedürfnisse als dringlicher und bedeutungsvoller erlebt werden. So sind beispielsweise Luft und Wasser essenzieller als ein teures Auto (ebd.).
} 


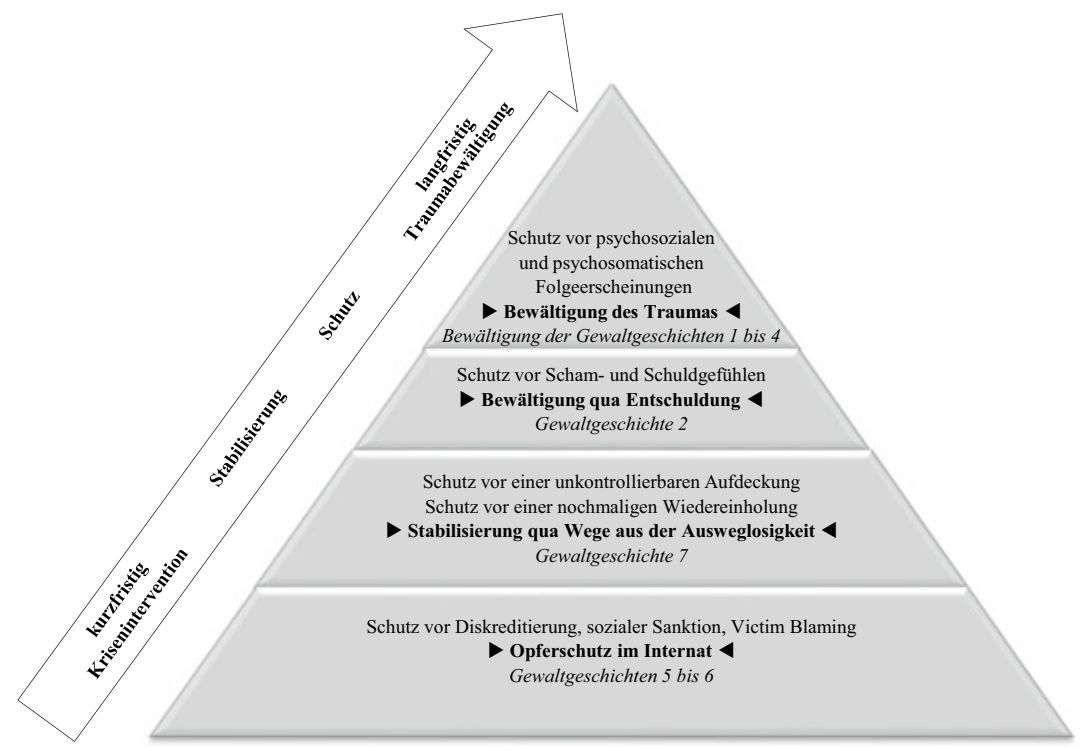

Abb. 8.1 Bedürfnishierarchie

Prozess das Gespräch mit Magdalena voraussetzt, sei exemplarisch eine fachlich naheliegende Bedürfnishierarchie skizziert (Abb. 8.1).

Folgt man den Schilderungen Magdalenas, belastet sie die Diskreditierung durch ihre Mitschüler*innen sowie die Ängste vor einer Aufdeckung und Wiedereinholung aktuell am stärksten. Demgemäß bedarf es zunächst des Schutzes vor weiterer (sexualisierter) Gewalt in der Peergruppe. Baut der Schutz vor weiterer Gewalt auf den Rückgewinn von Kontrolle und Handlungsmacht, ist dies zugleich einer Stabilisierung hinsichtlich ihrer Ängste zuträglich. Sobald Magdalena ausreichend stabilisiert wurde, erscheint eine Annäherung an die verspürten Scham- und Schuldgefühle angemessen. Von dort aus kann sukzessive zu (reaktivierten) Belastungen als Folgeerscheinung(en) vergangener Gewalterfahrungen gearbeitet werden. Dies umfasst das Schaffen einer Lebensumwelt, die die Risiken einer Reaktivierung und Retraumatisierung minimiert. Selbstverständlich kann trotz der visuellen Hierarchisierung eine punktuelle Gleichzeitigkeit von Interventionen fachlich sinnvoll sein. Beispielsweise können Interventionen zu Magdalenas Ängsten parallel zur institutionellen Aufarbeitung im Internat 
stattfinden. Dennoch sollte die Arbeit mit einer Bedürfnishierarchie zu einer diesbezüglichen kritisch-reflexiven Auseinandersetzung anregen: $\mathrm{Ob}$ und wie kann beispielsweise eine Stabilisierung gelingen, wenn mit Magdalena vorwiegend an dem sexuellen Missbrauch gearbeitet würde? Helfende stehen hierbei vor der Herausforderung, an den Bedürfnissen bzw. Schutzbedarfen entlang der einzelnen Gewaltgeschichten zu arbeiten und gleichzeitig die Bewältigung der anderen Gewaltgeschichten mitzudenken. So könnte etwa das Risiko bestehen, dass Magdalena im Zuge der Angstbewältigung erarbeitete Schutzräume im Prozess der Bewältigung des sexuellen Missbrauchs zu hinterfragen beginnt. Deshalb obliegt Helfenden eine Mitverantwortung, dahingehende Eventualitäten nach bestem Wissen und Gewissen zu berïcksichtigen.

\subsubsection{Opferschutz im Internat}

Für den Schutz vor (sexualisierter) Gewalt und Diskreditierung in der Klasse bzw. im Internat ist primär die Institution verantwortlich. Mit der Einführung des Bundeskinderschutzgesetzes im Jahr 2012 verstehen sich Lehrer*innen als Teil der Verantwortungsgemeinschaft. Infolgedessen obliegt dem Internat die Aufgabe, die Gewalt zu beenden und institutionell aufzuarbeiten. Wie dies gelingen kann, sei im Folgenden skizziert.

1. Bedürfnisorientierung als Handlungsmaxime

Im Sinne der systemisch-konstruktivistischen Lebensweltorientierung ist die Maxime der Orientierung an den Bedürfnissen der Adressat*innen gefordert. Für die Umsetzung institutioneller Maßnahmen des Opferschutzes bedeutet dies, Magdalena in höchstmöglichem Maße partizipieren zu lassen. Das umfasst das gemeinsame Besprechen von Maßnahmen einschließlich potenzieller Konsequenzen.

2. Einbezug einer externen Fachstelle, Benennen von Fallverantwortlichen In Fällen sexualisierter Peergewalt muss von einer größeren Zahl an Gewaltausübenden und Gewaltzeug*innen ausgegangen werden. Daher ist zu berücksichtigen, dass unterschiedlichen Personengruppen (z. B. Peers, Eltern, Pädagog*innen sowie die institutionelle Leitungsebene) Unterstützung zu offerieren ist. Grundsätzlich bietet sich eine beratende Begleitung durch eine externe Fachstelle an.

3. Die Widerfahrnisse Magdalenas fachlich adäquat einordnen

Um dem Schutzauftrag gegenüber Magdalena gerecht $\mathrm{zu}$ werden, bedarf es einer korrekten Einordnung der Situation. In der Fallvignette wird die 
Diskreditierung infolge der Verbreitung von Nacktfotos offenkundig als „Mobbing" bewertet, wodurch die sexualisierenden Momente der Beleidigung unzureichend beachtet werden (Kärgel \& Vobbe, 2020). Hierin liegt eine Bagatellisierungsgefahr sowohl bei der nicht konsensuellen Verbreitung sexualisierter Fotoaufnahmen in Jugendgruppen im Allgemeinen wie auch im Falle Magdalenas im Speziellen (Vobbe, 2014; Böhm et al., 2018). Gründe hierfür sind unter anderem fehlende Standards zur Bestimmung mediatisierter sexualisierter Gewalt (s. Kap. 4) sowie ihrer Abgrenzung zu angrenzenden Phänomenen wie Mobbing. Es bestehen zwar unbestreitbar Schnittmengen, etwa der Einsatz digitaler Medien, doch liegt das wesentliche Differenzierungsmerkmal in der sexistischen oder sexualisierenden Integritätsverletzung. Nach der Definition mediatisierter sexualisierter Gewalt (s. Kap. 2) handelt es sich bei der Verbreitung sexualisierter Fotos- und Videoaufnahmen sowie sexistisch-sexualisierter Diskreditierung mindestens um eine beabsichtigte sexualisierte Grenzverletzung. Ungeachtet dessen liegen unter Umständen Straftatbestände vor. Nach $\S$ 201a StGB ist beispielsweise das Herstellen und Verbreiten von Foto- und Videoaufnahmen, die den höchstpersönlichen Lebensbereich und/oder Persönlichkeitsrechte verletzen, strafbar. Nach § 184b StGB sind der Besitz und die Verbreitung kinder- und jugendpornografischer Inhalte ${ }^{6}$ strafbar und es kommt gegebenenfalls ein sexueller Missbrauch zulasten Annas infrage. Zu beachten ist überdies, dass das Strafmündigkeitsalter mit 14 Jahren beginnt. Demnach könnten sich auch Magdalenas Mitschüler*innen strafbar gemacht haben.

4. Maßnahmen des Opferschutzes

Entgegen der Bedeutsamkeit des „pädagogisch Methodischen“ zur Aufarbeitung von Mobbing verändern sich die Handlungsmaxime in Fällen sexualisierter Peergewalt „unmittelbar hin zur akuten Krisenintervention.“ (Vobbe, 2014, S. 204). Hieraus leiten sich nach Vobbe (ebd.) folgende Maßnahmen ab:

\section{Eine räumliche Trennung von Magdalena und den Gewaltausübenden initiieren}

Wesentlich ist dabei, dass nicht Magdalena der Eindruck entsteht, sie müsse den Klassenverband verlassen, sondern Sorge für ihren institutionellen Schutz vor den Gewaltausübenden getragen wird. Diesen Schutz faktisch herzustellen, ist kompliziert, da unterschieden werden muss zwischen Initiator*innen und

\footnotetext{
${ }^{6}$ Zur Definition, Einordnung und Identifikation kinder- und jugendpornografischer Inhalte findet sich Näheres in Kap. 10.
} 
Bystandern. Dies kann in einer Spannung dazu stehen, dass die Schule eigentlich keine Ermittlungsaufgaben übernehmen soll. Hinzu kommt, dass schulische Interventionen in Einklang mit landesunterschiedlichen Schulgesetzen und in Abstimmung mit den zuständigen Regierungspräsidien umzusetzen sind. Weitere mediatisierte Übergriffe können nicht gänzlich ausgeschlossen werden. Die Herstellung von räumlichem Schutz als Verantwortung der Schule ist deswegen auch hinsichtlich ihrer Symbolik und Signalwirkung für Magdalena zu verstehen. Möglichkeiten dazu sind, Gewaltausübende (vorübergehend) zu suspendieren, einen Klassenwechsel zu initiieren oder sie der Schule zu verweisen.

\section{Keine klärenden Gespräche in der Krisenintervention}

Ein Hinwirken auf klärende Gespräche zwischen Magdalena und den Gewaltausübenden ist ebenso zu vermeiden wie eine Beteiligung der Gewaltausübenden an Wiedergutmachungen. Es besteht andernfalls das Risiko, dass sie den Druck auf Magdalena fortsetzen oder Magdalena glaubt, ihre Verarbeitung bestehe in einer Entschuldigung der Gewalt.

\section{Eine unkontrollierbare Aufdeckung vermeiden}

Es besteht keine Notwendigkeit, gegenüber dem Internat den sexuellen Missbrauch durch Anton aufzudecken. Eine Einordnung als sexualisierte Peergewalt ist zur Gewährleistung adäquaten Opferschutzes ausreichend. Allerdings empfiehlt es sich, gemeinsam mit Magdalena eine Sprachregelung hinsichtlich des Entstehungskontexts der Nacktfotos zu erarbeiten.

5. Inverantwortungnahme der Gewaltausübenden als pädagogische Maßnahme Das Ziel pädagogischer Maßnahmen muss zuvorderst sein, Magdalena im Klassenverband zu rehabilitieren. Dazu gehören auch Maßnahmen der Inverantwortungnahme der Gewaltausübenden. Dies umfasst neben pädagogischendisziplinarischen Sanktionen (z. B. Suspendierung, Klassenwechsel, Schulverweis) und eventuellen rechtlichen Schritten auch eine Ansprache der Gefährder*innen. Eine solche richtet sich an jene Gewaltausübenden, die in den Rollen der Assistierenden oder Verstärkenden zur Fortführung der sexualisierten Gewalt beitragen. Das Ziel besteht in der Aufklärung und Sensibilisierung für das Gewalthandeln einschließlich dessen (potenzieller) juristischer Konsequenzen. Demgemäß wäre Magdalenas Mitschüler*innen darzulegen, dass das Weiterleiten von Nacktfotos als sexualisierte Peergewalt zu bewerten ist, die gegebenenfalls einen Straftatbestand darstellt und als solcher geahndet werden 
kann. In diesem Zusammenhang ist darüber zu informieren, dass das Vorwarnen von Personen, die ebenfalls im Besitz der Nacktfotos sein könnten oder diese womöglich bereits verbreitet haben, zusätzlich nach § 258 StGB als Strafvereitelung rechtlich sanktionierbar ist. Eine im HUMAN-Projekt interviewte Schulsozialarbeiterin kooperierte zur Ansprache von Gefährder*innen im Klassenverband erfolgreich mit der örtlichen Polizeibehörde. Eine Kooperation zwischen Schule und der Kinder- und Jugendhilfe ist ferner geraten, um individuelle sekundärpräventive Angebote der Täter*innenarbeit zu implementieren. Weitgehend unerforscht ist die Frage, wie Magdalena durch gruppenbezogene Solidarität und Empathie der Klassengemeinschaft sozial reintegriert werden kann. Insofern ist das Anwendungswissen spezialisierter Fachberatungsstellen gefordert.

\subsubsection{Wege aus der Ausweglosigkeit erarbeiten, Schutzräume schaffen}

Die skizzierten Maßnahmen des unmittelbaren Opferschutzes stellen zwar eine notwendige, jedoch keine hinreichende Bedingung einer langfristigen Stabilisierung dar. Angesichts der verspürten Ohnmacht sowie der erlebten Ängste bedarf es einer begleitenden Krisenintervention. Das Schaffen von Schutz ist in mediatisierten Gewaltkontexten insofern herausfordernd, als das Risiko einer (nochmaligen) Wiedereinholung fortwährend besteht. Schutz kann unseres Erachtens demnach in erster Linie das subjektive Empfinden von Kontrolle, Handlungsfähigkeit und Selbstbestimmung trotz ebendieses Risikos bedeuten. Das Ziel von Intervention(en) liegt folglich im Rückgewinn von Handlungsmacht und (Informations-)Kontrolle sowie im Schaffen von Alltagsroutinen, die das Risiko von Reaktivierung, Retraumatisierung und alltagsbegleitenden Ängsten minimieren. Hierfür bieten sich Interventionsmaßnahmen an, die sich an instrumentellen und emotionsgerichteten Strategien der Bewältigung belastender Lebensereignisse orientieren. Während eine emotionsgerichtete Bewältigung darauf abzielt, Ängste und Belastungen auf einer kognitiv-emotiven Ebene zu beschwichtigen oder abzubauen, stellen instrumentelle Bewältigungsstrategien auf einer Handlungsebene den Versuch dar, eine verspürte Belastung oder Bedrohung durch aktives Handeln zu minimieren bzw. zu beseitigen (Dehne, 2017; Goleman, 1991). Instrumentelle und emotionsgerichtete Bewältigungsstrategien sind dabei nicht gegeneinander abzuwägen. Im Gegenteil führt eine Kombination zu positiven Wechselwirkungen, die im Folgeverlauf aufgezeigt werden. 


\subsubsection{Instrumentelle Interventionsmöglichkeiten}

In Fällen der Wiedereinholung bezieht sich das Schaffen von Schutz durch aktives Handeln hauptsächlich auf Maßnahmen, die die Wahrscheinlichkeit einer Wiedereinholung verringern oder zumindest deren Ausmaß eindämmen. Hierfür bestehen verschiedenerlei technische und juristische Möglichkeiten.

\section{Das Löschen der Nacktfotos veranlassen}

Erstens gibt es Möglichkeiten, Foto- und Videoaufnahmen aus dem Internet zu entfernen bzw. entfernen zu lassen. Hierfür bedarf es jedoch einer entsprechenden fachlichen Expertise. Als erste Anlaufstelle empfehlen wir jugendschutz.net, „das gemeinsame Kompetenzzentrum von Bund und Ländern für den Schutz von Kindern und Jugendlichen im Internet“, das ,,mit gesetzlichem Auftrag [arbeitet], der im Jugendmedienschutz-Staatsvertrag niedergelegt ist.“ (Glaser, 2021). Das Kompetenzzentrum jugendschutz.net nimmt mit Anbieter*innen Kontakt auf, um Rechtsverstöße im Internet zu beseitigen und eine Benachteiligung von Kindern und Jugendlichen möglichst zu begrenzen. Im Jahr 2019 wurde bei Darstellungen sexualisierter Gewalt in 90 \% der Fälle Erfolg erzielt (Glaser, 2019).

\section{Rechtliche Ansprüche geltend machen}

Um einer abermaligen Veröffentlichung oder Verbreitung vorzubeugen, bestehen folgende Möglichkeiten zur Geltendmachung rechtlicher Ansprüche:

1. Geltendmachung eines Unterlassungsanspruchs

Laut $\S 823$ Abs. 1, 1004 Abs. 1 S. 2 BGB besteht bei einer Verletzung des allgemeinen Persönlichkeitsrechts ein Unterlassungsanspruch sowie ein Anspruch auf Schadensersatz. Da das allgemeine Persönlichkeitsrecht unter anderem den Schutz des Namens und der persönlichen Ehre umfassen, greift der Unterlassungsanspruch sowohl bei Beleidigung wie auch bei der unbefugten Verbreitung von Foto- und Videoaufnahmen sowie gespeicherten Kommunikationsverläufen, insbesondere wenn dies auch noch eine Straftat darstellt. Ein Unterlassungsanspruch kann auch Minderjährigen gegenüber geltend gemacht werden.

2. Geltendmachung von Löschungs- und Herausgabeansprüchen

Nach § 985 BGB kann die/der Eigentümer*in von der/dem Besitzer*in die Herausgabe der Sache, z. B. gespeicherte Medieninhalte, verlangen. In einem Urteil des Bundesgerichtshofs vom 13. Oktober 2015 (VI ZR 271/14) wird der Anspruch auf Löschung von Fotos nach dem Beenden einer Beziehung bejaht, sofern die Einwilligung zur Nutzung nur zeitlich begrenzt für die Dauer der Beziehung erteilt war. 
Die skizzierten Rechtsansprüche sind exemplarisch zu verstehen, da rechtliche Handlungsmöglichkeiten grundsätzlich fallabhängig sind. Deshalb raten wir ausdrücklich zu einer Rechtsberatung. Eine Übersicht über Anwält*innen mit einem Schwerpunkt in der Vertretung Gewaltbetroffener findet sich in der Datenbank „Hilfeportal Sexueller Missbrauch.“7

\section{Strafanzeige erstatten}

Eine weitere Option ist das Stellen einer Strafanzeige. Das Erstatten einer Strafanzeige ist Magdalena zwar trotz Minderjährigkeit ohne elterliches Einverständnis möglich, das Stellen eines Strafantrags, der jedenfalls für die Verfolgung einer Straftat nach § 201a StGB grundsätzlich notwendig ist, muss hingegen nach § 77 Abs. 3 StGB durch die Eltern erfolgen. Falls Magdalena eine Strafanzeige in Erwägung zieht, sie ihre Eltern jedoch in naher Zukunft nicht einweihen möchte, bietet es sich in Anbetracht der nahenden Volljährigkeit an, diese abzuwarten. Allerdings kann dann eine Verfolgung wegen § 201a StGB aufgrund fehlenden rechtzeitigen Strafantrags (Antragsfrist 3 Monate nach Kenntnis der Tat) scheitern.

1. Strafanzeige gegen Anton (mögliche einschlägige Tatbestände: $§ 174,176,177$, $182 \mathrm{StGB})$

Nach $\S 177$ StGB wird aufgrund sexueller Übergriffe, sexueller Nötigung oder Vergewaltigung bestraft, ,,wer gegen den erkennbaren Willen einer anderen Person sexuelle Handlungen an dieser Person vornimmt oder von ihr vornehmen lässt oder diese Person zur Vornahme oder Duldung sexueller Handlungen an oder von einem Dritten bestimmt." Je nach Näheverhältnis zu Anton als Trainer und dem Entwicklungsstand von Magdalena zum Zeitpunkt der Beziehung könnte eine Strafbarkeit wegen sexuellem Missbrauch Schutzbefohlener gemäß $\S 174$ Abs. 1 Nr. 1 StGB in Betracht kommen oder auch nach $\S 182$ Abs. 3 StGB wegen sexuellem Missbrauch Jugendlicher. Nach § 176 StGB wird wegen sexuellen Missbrauchs von Kindern bestraft, wer ein Kind (hier Anna) „dazu bestimmt, sexuelle Handlungen an einem Dritten vorzunehmen oder von einem Dritten an sich vornehmen zu lassen (Abs. II) oder an sich selbst vorzunehmen (sog. ,Posen“, Abs. IV Nr. 2). Überdies kommt eine Strafbarkeit Antons wegen unbefugten Verbreitens intimer Bildaufnahmen nach § 201a Abs. 1 Nr. 4 StGB

\footnotetext{
${ }^{7}$ Das „Hilfeportal Sexueller Missbrauch“ ist eine Datenbank, in der örtliche Beratungsstellen und andere Unterstützungseinrichtungen (Ärzt*innen, Psychotherapeut*innen, Anwält*innen) aufgelistet werden, die passgenaue Angebote für Betroffene sexualisierter Gewalt, deren Angehörige und Fachkräfte bereithalten: https://beauftragter-missbrauch.de/ hilfe/beratung-und-hilfe (Arbeitsstab des UBSKM 2020).
} 
und wegen Herstellens kinder- oder jugendpornografischer Inhalte gemäß § 184 $\mathrm{b}$ und $\mathrm{c}$ StGB in Betracht.

2. Strafanzeige gegen die Verbreitenden und Besitzenden $(\S 184,184 \mathrm{~b}, 184 \mathrm{c}, 201 \mathrm{a}$ StGB)

Mit § 201a StGB ist die Verbreitung von Bildaufnahmen, die den höchstpersönlichen Bereich einer Person betreffen (z. B. Nacktheit), strafbar. Nach § 184 StGB ist das Zugänglichmachen pornografischer Inhalte an Minderjährige und nach §§ $184 \mathrm{~b}$ und c StGB sind sowohl der Besitz wie auch die Verbreitung kinder- und jugendpornografischer Inhalte strafbar. Dies setzt allerdings die Identifikation entsprechender Personen voraus. In Magdalenas Fall dürfte es im Rahmen polizeilicher Ermittlungsverfahren möglich sein, die Verbreitenden und Besitzenden innerhalb des Klassenchats zu identifizieren. Davon ausgehend, dass Magdalenas Mitschüler*innen das vierzehnte Lebensjahr überschritten haben, sind sie nach $\S 19$ StGB und § 1 JGG bedingt strafmündig.

Während Magdalena zur selbstbestimmten Entscheidungsfindung die Möglichkeit zur Beratung mit spezialisierten Fach- bzw. Opferanwält*innen erhalten sollte, empfiehlt es sich im Rahmen der Information und Aufklärung, auf mögliche Konsequenzen einer Strafanzeige respektive eines Verzichts zu verweisen.

3. Juristische Konsequenzen aufgrund der Verwicklung Annas

Zweifelsohne trifft Magdalena keine moralische Schuld an der sexualisierten Gewalt gegen Anna. Unter juristischen Gesichtspunkten kann angesichts ihrer Strafmündigkeit eine Täterinnenschaft nicht ausgeschlossen werden. Nach § 25 Abs. 1 Alt. 2 StGB kommt zusätzlich Anton als mittelbarer Täter in Betracht. Dies ist der Fall, wenn ein Hintermann sich eines ,menschlichen Werkzeugs“ bedient und dabei die Tatherrschaft innehat. Ein*e mittelbare*r Täter*in begeht die Tat durch eine*n anderen, sie*er verwirklicht die Tatbestandsmerkmale nicht oder nicht ganz selbst, sondern bedient sich zusätzlich einer anderen Person, kraft überlegenen Wissens oder kraft überlegenen Wollens. Dies verhindert allerdings nicht die Strafbarkeit der Person, derer er sich bedient. Ebenfalls in Betracht kommt, dass Anton nach $\S 26$ StGB jedenfalls als Anstifter bestraft werden könnte, womit aufgrund der gerade eingetreten Strafmündigkeit Magdalenas durchaus wahrscheinlich wäre, dass ein Verfahren gegen sie eingestellt würde oder allenfalls eine Verwarnung ausgesprochen würde.

4. Konsequenzen für Anna

Durch eine Strafanzeige wird Anna unweigerlich mit ihren zurückliegenden Missbrauchserfahrungen konfrontiert. Da sie kein Zeugnisverweigerungsrecht hat, wäre sie damit eventuell gegen ihren Willen in ein Strafverfahren und zu einer Zeugenaussage gezwungen. In Anbetracht ihrer Minderjährigkeit ist hierbei eine Aufdeckung gegenüber Annas Eltern unvermeidbar. Anna selbst wollte 
diese Aufdeckung vor drei Jahren. Wie ihre Situation aktuell ist, wissen wir nicht. Eine Einbeziehung Annas in die Entscheidung wäre insofern sinnvoll und es können entsprechende Unterstützungsangebote initiiert werden. Durch eine Anrufung des Jugendamts kann dafür Sorge getragen werden, dass eine Vernehmung durch die Kinder- und Jugendhilfe unterstützt wird und sowohl Anna wie auch ihre Eltern adäquate Unterstützungsangebote erhalten.

5. Risiko der Aufdeckung gegenüber Magdalenas Eltern

$\mathrm{Da}$ in einen Strafprozess sowohl Anna wie auch ihre Erziehungsberechtigten involviert wären, bestünde das Risiko einer Aufdeckung gegenüber Magdalenas Eltern auch im Falle einer Strafanzeige ohne elterliche Kenntnis.

6. Risiko einer Reaktivierung und Potenzierung von Belastungen Ein Strafprozess kann zu einer Reaktivierung von Schuldgefühlen, Gefühlen des Verrats gegenüber Anna (Allroggen et al., 2016) oder aber einer Verstärkung der Angst vor Aufdeckung und den sozialen Folgen einer Aufdeckung führen.

7. Beweissicherung

Das Erstatten einer Strafanzeige geht mit dem Sichern von Beweisen einher. Für Gewaltbetroffene bedeutet das zumeist, dass ihr Smartphone und gegebenenfalls weitere mobile Endgeräte (z. B. Tablet, Notebook) für einige Tage (oder auch länger) bei der Polizei verbleiben. Hierüber ist Magdalena im Vorfeld aufzuklären. Schließlich ist es denkbar, dass dort Telefonnummern oder Inhalte gespeichert sind, die für das Mädchen eine Ressource darstellen, die alsdann für einen bestimmten Zeitraum nicht verfügbar wäre. Diese Information kann allerdings dazu führen, dass Magdalena schambehaftete „Beweismaterialien“ vernichten möchte. Ein dahingehendes Risiko ist bei Ängsten vor einer eigenen Strafbarkeit umso höher. Entscheidet sich Magdalena gegen das Erstatten einer Strafanzeige, sollte sie erfahren, dass eine Beweissicherung, also die Sicherung der Aufnahmen und gegebenenfalls von Hinweisen auf deren Verbreitung, dennoch sinnvoll sein kann, um ihr auch zu einem späteren Zeitpunkt die Möglichkeit zur Strafanzeige zu gewähren, ohne Gefahr zu laufen, dass Dritte sämtliche Beweismaterialien vernichteten.

\section{Die Auffindbarkeit verbreiteter Nacktfotos erschweren}

Zuletzt sei auf eine Möglichkeit verwiesen, mittels derer eigeninitiativ die Auffindbarkeit veröffentlichter Foto- und Videoaufnahmen in sozialen Netzwerken und Suchmaschinen erschwert wird. Sind die Schlagworte bekannt, die mit den verbreiteten Medieninhalten verknüpft sind, kann man sich dieses Wissen strategisch zunutze machen. So ist es möglich, unter demselben Schlagwort eine Flut anderer Fotos oder Videos zu posten. Dadurch minimiert sich die Wahrscheinlichkeit, dass die unerwünschten Foto- oder Videoaufnahmen von Dritten gefunden werden. 
Dieser Vorschlag wurde im Rahmen der Focus Group Interviews von einem Sozialarbeiter (Sexualpädagogik und -beratung) eingebracht, der hiermit gute Erfahrungen sammelte.

\begin{abstract}
„Also, ich hatte gerade auch die Fantasie zu schauen, welche Schlagwörter das sind, mit denen man das Bild findet und dann sozusagen über eine verschiedene Flut von weiteren Bildern, die gepostet werden unter dem Schlagwort, verliert es ja auch seine Bedeutungsschwere sozusagen im digitalen Raum, weil es gar nicht mehr so einfach zu finden ist. [...] Also wir haben halt in der praktischen Arbeit über verschiedene Schlagwörter/ also eher per Zufall habe ich das entdeckt, habe mir Hashtags gemacht und da kommen super viele Bilder jetzt einfach von unserem Experiment-Projekt, wo es halt um Fotos ging.“
\end{abstract}

Sozialarbeiter, Sexualpädagogik und -beratung, FGI

\title{
8.2.3.2 Emotionsgerichtete Interventionsmöglichkeiten
}

Emotionsgerichtete Interventionen setzen zwar auf einer kognitiv-emotiven Ebene an, um Belastungen abzubauen (Dehne, 2017; Goleman, 1991), doch wirken sie auf die Handlungsebene ein. Durch das angeleitete Antizipieren der Bedeutsamkeit und Folgeschwere verschiedenartiger Handlungsszenarien kann ein Reflexionsprozess angestoßen werden, der das Handlungsspektrum Magdalenas unter Berücksichtigung ihrer Lebenswelt, Wünsche und Bedürfnisse erweitert. Beispielsweise könnte eine geringere Angst vor der elterlichen Reaktion (kognitiv-emotive Ebene) den Einbezug der Eltern (Handlungsebene) ermöglichen. Dementsprechend erfüllen emotionsgerichtete Interventionen dreierlei Funktionen. Erstens ermöglichen sie es Helfenden, Magdalenas Lebenswelt einschließlich ihrer Perspektivität, Belastungen und Bedürfnisse zu verstehen und den Handlungsrahmen der Intervention(en) entsprechend herauszuarbeiten. Zweitens sind sie der Bewältigung dienlich, u. a. indem sie drittens das Handlungsspektrum instrumenteller Intervention(en) erweitern. Das Ziel ist es demnach, Magdalena dabei zu unterstützen, das Risiko einer erneuten Verbreitung und damit verbundener Folgen als Teil ihres Lebens zu verstehen, der in ihr Lebensskript eingewebt werden muss. Im Sinne des Schutzes geht es also darum, Magdalena hinsichtlich ihrer Handlungsfähigkeit und (Informations-)Kontrolle im Falle sich bewahrheitender Ängste zu bestärken und befähigen. Hierfür steht eine Reihe möglicher Interventionen zur Verfügung, die sich an den Grundsätzen der systemischen Therapie und Beratung sowie therapeutischer Ansätze orientieren. Die nachfolgende exemplarische Auswahl begrenzt sich auf diejenigen 
Ansätze, die in ihrem Kern der Maxime einer systemisch-konstruktivistischen Lebensweltorientierung entsprechen.

\section{Bewertungsmaßstäbe hinterfragen lernen und Handlungsmöglichkeiten ent- decken durch Systemisches Fragen ${ }^{8}$}

Systemisches Fragen bezeichnet nach Patrzek (2017) jene Fragetechniken, die einem systemischen Verständnis folgend perspektivische Vielfalt begünstigen und letztendlich den individuellen Entscheidungs- und Handlungsspielraum vergrößern. Demgemäß bedeutet systemisches Fragen, die Konstruktion der Lebenswelt zu thematisieren, zu hinterfragen und gegebenenfalls zu verändern, sich vorhandene Ressourcen zu vergegenwärtigen und deren Aktivierungen anzustoßen. Hierfür bieten sich in der Arbeit mit Magdalena insbesondere die folgenden Fragetechniken an.

\section{Hypothetische Fragen}

Hypothetische Fragen führen in Form von ,Wenn-dann-Szenarien“ fiktive Situationen in das Gespräch ein und erfragen auf diese Weise denkbare Reaktionen von sich oder anderen Personen. Dieserart könnte beispielsweise herausgefunden werden, wie Magdalena a) zum Einweihen der Eltern, b) dem Erstatten einer Strafanzeige, c) der Unterbringung in einer Einrichtung der stationären Kinder- und Jugendhilfe, d) einer Aufdeckung gegenüber unterschiedlichen Bezugssystemen oder aber e) einer erneuten Verbreitung steht.

2. Zirkuläre Fragen fokussieren auf die vermutete Fremdperspektive, ,weil das Verhalten von Menschen nicht von dem bestimmt wird, was andere tatsächlich über sie denken, sondern von dem, was sie denken, was andere denken." (Simon \& Simon, 2006, S. 223). In Zusammenhang mit Magdalenas Bewertung, die Eltern einzuweihen oder eine Strafanzeige zu stellen, ist die Ergänzung hypothetischer Fragen um zirkuläre Fragen insofern relevant, als Magdalenas Ängste vor den sozialen Folgen für Beratende und Helfende verstehbar werden und das Mädchen einen reflektorischen Zugang zu alternativen Denk- und Betrachtungsweisen erhält. Durch zirkuläre Fragen können beispielsweise

- die vermutete elterliche Bewertung einer Aufdeckung,

- die vermutete elterliche Bewertung der Rolle Magdalenas bei der Verwicklung Annas,

\footnotetext{
${ }^{8}$ Eine detaillierte Übersicht über systemische Fragetechniken und deren Anwendung finden sich einschließlich anschaulicher Beispiele in der Monografie „Systemisches Fragen. Professionelle Fragetechnik für Führungskräfte, Berater und Coaches" von Patrzek oder aber in dem Übersichtskapitel ,Systemische Beratung und der systemische Ansatz in der Sozialen Arbeit“ von Haselmann, das in dem Sammelband „Methodenbuch Soziale Arbeit“ von Michel-Schwartze erschien.
} 
- die vermutete Perspektive Annas auf das Stellen einer Strafanzeige,

- die vermutete Bewertung jener Mitschüler*innen, die sich nicht aktiv an Magdalenas Diskreditierung beteiligten,

erarbeitet werden.

\section{Perspektivwechsel und Wege aus der Ausweglosigkeit durch Zukunftsprojek- tionen ${ }^{9}$}

Mittels Antizipation werden in der Methode der Zukunftsprojektionen nach Mahr (2018) alte Lebensentwürfe neuen Lebensentwürfen gegenübergestellt. Der mehrperspektivische Blick in die Zukunft erleichtert es Adressat*innen, Auswege aus ihren als belastend oder dilemmatisch erlebten Situationen zu erarbeiten. In einem ersten Schritt geht es darum, die bisherigen Konsequenzen von Magdalenas Umgang mit ihren Gewaltgeschichten zu reflektieren:

- Was wäre heute anders, wenn du damals (Gewaltgeschichten 1 bis 4) keine Angst vor den Folgen einer Strafanzeige gehabt hättest?

- Was wäre heute anders, wenn du den Sportverein nicht verlassen hättest?

In einem zweiten Schritt werden mögliche Folgen neuer Überzeugungen herausgearbeitet:

- Was, glaubst du, wird sich in deinem Leben durch z. B. eine Strafanzeige verändern?

Anschließend werden die möglichen Lebensentwürfe umfassend und getrennt voneinander bearbeitet und antizipiert. Auf dieser Grundlage erhält Magdalena den Raum, in die Zukunft zu blicken:

- Woran hat dich z. B. die Entscheidung gegen eine Aufdeckung gehindert?

- Wie viel Freude hast du aufgrund dieser Entscheidung(en) verloren?

- Inwieweit haben diese Entscheidungen deine sozialen Beziehungen beeinflusst?

- Was lösen diese Fragen in dir aus?

- Was könnte heute ohne diese Entscheidung anders sein?

\footnotetext{
${ }^{9}$ Möglichkeiten zur Umsetzung der Methode der Zukunftsprojektionen finden sich als Überblick im „Praxishandbuch Integrative Psychotherapie“ von Mahr.
} 
Nachdem Magdalena die gedankliche Reise abschüttelt, malt sie sich unter Anleitung ihre alternative Zukunft aus:

- Inwiefern hat die Entscheidung für eine Aufdeckung dein Leben bereichert?

- Wie fühlst du dich damit?

- Was könntest du jetzt zu dir selbst sagen, was du dich zuvor (noch) nicht getraut hast?

Sofern Magdalena einen alternativen neuen Lebensentwurf als aussichtsvoll und entlastend erlebt, ist sie dahingehend zu befähigen.

\section{Entscheidungs- und Handlungsdilemmata durch die Arbeit mit Stühlen ${ }^{10}$ auflösen}

Mithilfe der Stuhltechnik, die ihren Ursprung im Psychodrama von Moreno hat und von Perls im Rahmen der Gestalttherapie weiterentwickelt wurde, werden innere Konflikte, Widersprüche oder konflikthafte soziale Dynamiken erfahrbar und verstehbar, indem sie als in einer belastenden Situation wirkende Kräfte (z. B. Personen, innere Konflikte) auf einem eigenen Stuhl platziert werden und durch die Adressat*innen eine Stimme bekommen. In verschiedenerlei therapeutischen Kontexten erwies sich die Arbeit mit Stühlen als besonders wirksam in der Auflösung von Ambivalenzen und Entscheidungsschwierigkeiten (Kellogg, 2011; Rosner \& Henkel, 2010). Daher empfehlen wir die Stuhltechnik in der Arbeit mit Magdalena, um das erlebte Entscheidungsdilemma zwischen ,Flucht“(Schul-/Wohnortwechsel) und „Ausharren bzw. Leiden“ zu lösen. Der Grobablauf gestaltet sich nach Mahr (2018) wie folgt: Zunächst schildert Magdalena ihre Ambivalenzen respektive ihr Entscheidungsdilemma. Anschließend werden die konfligierenden Perspektiven bzw. Gefühle auf zwei Stühlen einander gegenüber positioniert. Magdalena erläutert unter Anleitung ihren Konflikt von einem Stuhl und damit aus einer Perspektive ihrer Wahl abermals. Nun wird der Konflikt nochmals vom anderen Stuhl aus dessen stellvertretender Perspektive dargelegt. Darauf folgt ein angeleiteter imaginärer Dialog zwischen Magdalena und der auf dem gegenüberliegenden Stuhl positionierten Konfliktseite. Durch ein mehrfaches Wechseln der Stühle wird eine intensive Auseinandersetzung mit dem inneren Konflikt bzw. dem Entscheidungsdilemma angeregt. In einer abschließenden Reflexion erhält sie den Raum, ihre gewonnenen Einsichten und Erkenntnisse zu reflektieren. Wenn es nicht ohnehin zu einer deutlich erkennbaren Klärung des Konfliktes gekommen ist, sollte sie gefragt werden,

\footnotetext{
${ }^{10}$ Möglichkeiten zur Umsetzung der Arbeit mit Stühlen finden sich als Überblick im „Praxishandbuch Integrative Psychotherapie“ von Mahr.
} 
was sich im Gegensatz zu vorher verändert hat und ob es jetzt eine stärkere Tendenz zu der einen oder anderen Position gibt.

\section{Sichere Orte als Schutzraum auf dem Weg zum Rückgewinn von Sicherheit und Kontrolle}

Als jederzeit aufsuchbare Rückzugsorte in Momenten besonderer Belastung haben sich sichere Orte in (trauma-)therapeutischen Kontexten als erfolgversprechende Intervention zur Stabilisierung bewährt (Reddemann, 2017). Stadler (2002) empfiehlt die Einführung bzw. das Schaffen eines sicheren Orts unter Zuhilfenahme der Mittel des Psychodramas. Der Vorteil bestehe darin, einen andernfalls rein imaginierten und verbalisierten sicheren Ort real einzurichten. Ob dieser sichere Ort auf der Hauptbühne oder einer Nebenbühne eingerichtet wird, sei den Adressat*innen überlassen. Das wesentliche Kennzeichen des sicheren Orts sei dabei seine Entwicklung und Integration in bedrohlichen Situationen. Auf diese Weise würden das Gefühl von Sicherheit bestärkt und Ressourcen dafür geschaffen, sich im Alltag geschützt zu bewegen. Nicht zuletzt deshalb sei es bedeutsam, dass der sichere Ort möglichst bildhaft und detailgetreu beschrieben wird und bereits zu Beginn definiert wird, wer Zugang zu dem sicheren Ort hat. Über ein durch die Adressat*innen ausgewähltes Symbol ist der sichere Ort begehbar. Über die Abfrage des Sicherheitsgefühls vor und nach Aufsuchen des sicheren Orts kann der Erfolg des Ansatzes überprüft werden. Gemäß unserem Wissensstand wurde der Ansatz des sicheren Orts jedoch im Kontext mediatisierter sexualisierter Gewalt weder ausreichend erprobt noch weiterentwickelt.

\subsubsection{Entschulden}

Die Schuldgefühle Magdalenas nehmen gewissermaßen eine Schlüsselrolle ein. Als maßgebende Ursache der Angst vor den sozialen Folgen einer Aufdeckung begrenzen sie unter Umständen die Handlungsmöglichkeiten, etwa das Stellen einer Strafanzeige oder den Einbezug der Eltern als Ressource und verstärken das Ohnmachtserleben. Umso wesentlicher ist eine gelingende Entschuldung, die jedoch voraussetzt, dass sich Magdalena selbst verzeiht. Hierbei kann sie in beraterischen wie therapeutischen Settings angeleitet und unterstützt werden. Schuldgefühle resultieren im vorliegenden Fall aus der Vermengung negativer Attribution (Tran, 2015) und dem Erleben des Leides, dass man anderen vermeintlich zugefügt hat (Baumeister, 1998). Im Prozess des sich Verzeihens geht es hauptsächlich um eine Auseinandersetzung mit Fragen danach, inwiefern sich 
Magdalena für die Verwicklung Annas verantwortlich fühlt und weshalb es für Betroffene meistens schwierig ist, Hilfe zu initiieren.

Über ein De- und Reframing der Rolle Magdalenas kann ihr Handeln neu kontextualisiert und eine Entschuldung bewirkt werden. Deframing bedeutet, eine Handlung zu entrahmen bzw. zu entkontextualisieren. In einem anschließenden Reframing wird die Handlung neu gerahmt bzw. kontextualisiert. Dahinter steht der Gedanke, dass die Bedeutung einer Handlung von dem Rahmen bzw. den Rahmenbedingungen abhängt, innerhalb derer sie wahrgenommen wird (Holzfuss, 2021). Es gilt zu verdeutlichen, dass das in einem bestimmten Kontext als problematisch empfundene Verhalten in einem anderen Kontext angemessen ist. Indem Magdalena durch Beratende oder Therapeut*innen einen Raum erhält, um zu reflektieren, aus welchen Motiven heraus und mit welchen Absichten sie handelte und um sich mit der Frage auseinanderzusetzen, inwieweit ihr Tun durch Anton beeinflusst wurde, wird der Prozess eines De- und Reframings automatisch angestoßen. ${ }^{11}$ Wenn Magdalena trotz Reframing zu der Einsicht gelangt, Fehler begangen zu haben, kann mir ihr gemeinsam - beispielsweise mittels zirkulärer Fragen - erörtert werden, ob ein gemeinsames klärendes Gespräch mit Anna vorstellbar wäre. Bereits die hierdurch übernommene Verantwortung für wahrgenommenes Fehlverhalten kann unabhängig von etwaigen Schuldvorwürfen durch Anna entlastend wirken (Hirsch, 2008). Ebenso kann es entlasten, durch Dritte - etwa Beratende, Angehörige oder aber die Justiz - von Schuld befreit zu werden. Gewaltbetroffene erleben eine richterliche Schuldbefreiung teils als entlastend. Dies soll und darf jedoch nicht der einzige Grund für eine Strafanzeige sein. Denn die mit einer Strafanzeige potenziell verbundenen Konsequenzen und Belastungen werden durch die Chance auf eine richterliche Entschuldung nicht notwendigerweise relativiert.

\section{Literatur}

Allroggen, M., Gerke, J., Rau, T., \& Fegert, J. M. (2016). Umgang mit sexueller Gewalt: Eine praktische Orientierungshilfe für pädagogische Fachkräfte in Einrichtungen für Kinder und Jugendliche. Ulm: Universitätsklinik. https://www.uniklinik-ulm.de/filead $\mathrm{min} /$ default/Kliniken/Kinder-Jugendpsychiatrie/Dokumente/Sprich_mit_Handlungsemp fehlungen.pdf.

Arbeitsstab des UBSKM. (2020). Beratungsstellen und Hilfe in der Nähe. https://beauftrag ter-missbrauch.de/hilfe/beratung-und-hilfe. Zugegriffen: 20. Dez. 2020.

11 Über die Methoden des De- und Reframings wird beispielsweise ausführlich in dem Werk „Reframing: Neurolinguistisches Programmieren und die Transformation von Bedeutung“ von Bandler und Grinder (2010) berichtet. 
Bandler, R., \& Grinder, J. (Hrsg.). (2010). Reframing: Neurolinguistisches Programmieren und die Transformation von Bedeutung (9. Aufl.). Junfermann.

Bange, D. (2015). Unterstützung für Bezugs- und Kontaktpersonen sexuell missbrauchter Kinder und Jugendlicher. In J. M. Fegert, U. Hoffmann, E. König, J. Niehues, \& H. Liebhardt (Hrsg.), Sexueller Missbrauch von Kindern und Jugendlichen (S. 274-284). Springer VS.

Baumeister, R. F. (1998). Inducing guilt. In J. Bybee (Hrsg.), Guilt and children (S. 127-138). Academic Press.

Baumeister, R. F. (2013). Vom Bösen: Warum es menschliche Grausamkeit gibt. Huber.

Böhm, M., Budde, J., \& Dekker, A. (2018). Sexuelle Grenzverletzungen mittels digitaler Medien an Schulen: Annäherung an einen doppelten Verdeckungszusammenhang. MedienPädagogik: Zeitschrift für Theorie und Praxis der Medienbildung (Occasional Papers): 1-18. doi: https://doi.org/10.21240/mpaed/00/2018.02.21.X.

Cooper, K., Quayle, E., Jonsson, L., \& Svedin, C. G. (2016). Adolescents and self-taken sexual images: A review of the literature. Computers in Human Behavior, 55, 706-716. https://doi.org/10.1016/j.chb.2015.10.003.

Dehne, M. (2017). Soziologie der Angst. Springer VS.

Dekker, A., Koops, T., \& Briken, P. (2016). Sexualisierte Grenzverletzungen und Gewalt mittels digitaler Medien: Zur Bedeutung digitaler Medien für Phänomene sexualisierter Grenzverletzungen und Gewalt gegen Kinder und Jugendliche. Arbeitsstab des Unabhängigen Beauftragten für Fragen des sexuellen Kindesmissbrauchs.

Diener, E. (2013). The remarkable changes in the science of subjective well-being. Perspectives on Psychological Science: A Journal of the Association for Psychological Science, 8(6), 663-666. https://doi.org/10.1177/1745691613507583.

Doll, D., \& Nagel, B. (2019). Erwartungen an Anerkennung nach sexueller Gewalt in der Kindheit und Implikationen für die Soziale Arbeit. Soziale Passagen, 11(2), 305-322. https://doi.org/10.1007/s12592-019-00326-0.

Döring, N. (2012). Erotischer Fotoaustausch unter Jugendlichen: Verbreitung, Funktionen und Folgen des Sexting. Zeitschrift für Sexualforschung, 25, 4-25.

Ehring, T. (2013). Behandlung der posttraumatischen Belastungsstörung bei erwachsenen Überlebenden sexueller oder körperlicher Gewalt in der Kindheit. In A. Maercker (Hrsg.), Posttraumatische Belastungsstörungen: Mit 40 Tabellen (4. Aufl., S. 399-418). Springer VS.

Glaser, S. (2019). Jugendschutz im Internet. Bericht 2019: Risiken und Handlungsbedarf. https://www.jugendschutz.net/fileadmin/download/pdf/bericht2019.pdf.

Glaser, S. (2021). Mehr Rücksicht auf Kinder und Jugendliche im Internet!: jugendschutz.net ... http://www.jugendschutz.net/ueber-jugendschutznet/. Zugegriffen: 27. Okt. 2020.

Goleman, D. (1991). Lebenslügen: Warum wir uns immer wieder selbst täuschen. Beltz Juventa.

Haselmann, S. (2007). Systemische Beratung und der systemische Ansatz in der Sozialen Arbeit. In B. Michel-Schwartze (Hrsg.), Methodenbuch Soziale Arbeit (S. 153-206). Springer VS.

Hirsch, M. (2008). Scham und Schuld - Sein und Tun. Psychotherapeut, 53(3), 177-184. https://doi.org/10.1007/s00278-008-0605-y.

Holzfuss, B. (2021). Deframing: NLP-glossar. https://www.nlp-ausbildung-holzfuss.de/nlpinformationen/nlp-glossar/88-deframing. Zugegriffen: 20. Dez. 2020. 
Jones, P. J., Bellet, B. W., \& McNally, R. J. (2020). Helping or harming? The effect of trigger warnings on individuals with trauma histories. Clinical Psychological Science, 8(5), 905917. https://doi.org/10.1177/2167702620921341

Kaiser, C. (2020). Gewalterfahrung im Lebensverlauf. Blätter der Wohlfahrtspflege, 167(4), 130-133. https://doi.org/10.5771/0340-8574-2020-4-130.

Kärgel, K., \& Vobbe, F. (2020). Mediatisierte Gewalt - Diffusion - Transzendenz: Erscheinungsformen und Herausforderungen sexualisierter Gewalt mit digitalem Medieneinsatz. Kindesmisshandlung und -vernachlässigung, 23(1), 30-43.

Kattermann, V. (2019). Täter-Opfer-Dynamiken nach sexuellem Missbrauch in der psychoanalytischen Ausbildung. Forum der Psychoanalyse, 35(4), 373-389. https://doi.org/10. 1007/s00451-019-00336-x.

Kellogg, S. (2011). Veränderungen durch Stühlearbeit (transformational chairwork): Eine Einführung in psychotherapeutische Dialoge. In E. Roediger (Hrsg.), Fortschritte der Schematherapie: Konzepte und Anwendungen (S. 74-85). Hogrefe.

Keupp, H., Mosser, P., Busch, B., Hackenschmied, G., \& Straus, F. (Hrsg.). (2019). Die Odenwaldschule als Leuchtturm der Reformpädagogik und als Ort sexualisierter Gewalt. Springer VS.

König, O. (2016). Macht in Gruppen: Gruppendynamische Prozesse und Interventionen. Klett-Cotta.

Kraus, B. (2006). Lebenswelt und Lebensweltorientierung. Eine begriffliche Revision als Angebot an eine systemisch-konstruktivistische Sozialarbeitswissenschaft. Zeitschrift für Systemische Therapie und Familientherapie, 37(2), 116-129.

Mahr, C. (Hrsg.). (2018). Praxishandbuch integrative psychotherapie. Springer VS.

Maslow, A. H. (1943). A theory of human motivation. Psychological Review, 50(4), 370-396. https://doi.org/10.1037/h0054346.

Merten, K. (1999). Gewalt durch Gewalt im Fernsehen? Springer VS.

Michel-Schwartze, B. (Hrsg.). (2007). Methodenbuch Soziale Arbeit. Springer VS.

Patrzek, A. (2017). Systemisches fragen. Springer VS.

Paul, C. (2016). Scham nach sexualisierter Gewalt in Kindheit und Jugend als Folge öffentlicher Zuschreibungen. Forum Gemeindepsychologie, 21(1), 1-13.

Reddemann, L. (2017). Imagination als heilsame Kraft: Ressourcen und Mitgefühl in der Behandlung von Traumafolgen (20. Aufl.). Klett-Cotta.

Rosen, L. H., DeOrnellas, K., \& Scott, S. R. (2017). Bullying in school. Palgrave Macmillan US.

Rosner, R., \& Henkel, C. (2010). Die Gestalttherapie in der Psychotraumatologie: Charakteristika und Wirksamkeit gestalttherapeutischer Interventionen bei Posttraumatischen Belastungsstörungen. Trauma \& Gewalt, 4(4), 1-11.

Ryff, C. D., \& Keyes, C. L. M. (1995). The structure of psychological well-being revisited. Journal of Personality and Social Psychology, 69(4), 719-727. https://doi.org/10.1037/ 0022-3514.69.4.719.

Salmivalli, C., Lagerspetz, K., Björkqvist, K., Österman, K., \& Kaukiainen, A. (1996). Bullying as a group process: Participant roles and their relations to social status within the group. Aggressive Behavior, 22(1), 1-15. https://doi.org/10.1002/(SICI)1098-2337(199 6)22:1\%3C1:AID-AB1\%3E3.0.CO;2-T. 
Schäfer, M., \& Korn, S. (2004). Bullying als Gruppenphänomen. Zeitschrift für Entwicklungspsychologie und Pädagogische Psychologie, 36(1), 19-29. https://doi.org/10.1026/ 0049-8637.36.1.19.

Schattenburg, L. (2011). Unerwartete heftige Reaktivierung traumatischer Erlebnisse. Psychotherapeut, 56(4), 337-340. https://doi.org/10.1007/s00278-011-0840-5.

Schulze, H., \& Zimmermann, P. (2012). Ein Ort, wo ich einfach sein kann. Sozial Extra, 36(5-6), 25-28. https://doi.org/10.1007/s12054-012-0058-y.

Simon, F. B., \& Rech-Simon, C. (2006). Zirkuläres Fragen: Systemische Therapie in Fallbeispielen: Ein Lernbuch (7. Aufl.). Carl-Auer-System.

Slepian, M. L., Kirby, J. N., \& und Kalokerinos, E. K. (2020). Shame, guilt, and secrets on the mind. Emotion, 20(2), 323-328. https://doi.org/10.1037/emo0000542.

Smokowski, P. R., \& Evans, C. B. R. (2019). Bullying and victimization across the lifespan. Springer International Publishing.

Stadler, C. (2002). Von Sicheren Orten und Inneren Helfern. Zeitschrift für Psychodrama und Soziometrie, 1(2), 177-186. https://doi.org/10.1007/s11620-002-0018-9.

Staub-Bernasconi, S. (2018). Soziale Arbeit als Handlungswissenschaft: Soziale Arbeit auf dem Weg zu kritischer Professionalität (2. Aufl.). Budrich.

Tran, T. A. (2015). Wie missbrauchsspezifische Attribution, Scham und Angst die Aufdeckung sexuellen Missbrauchs in der Kindheit verzögern und sich pathopsychologisch auswirken: Das Interaktive ASA-Modell/ How abuse-specific attribution, shame and anxiety delay disclosure of child sexual abuse and promote pathopsychological developments: The Interactive ASA-Model. Kindesmisshandlung und-vernachlässigung, 18(1), 44-63.

Vobbe, F. (2014). Notwendige Differenzierungen im Umgang mit Bullying und sexualisierter Peergewalt. Kindesmisshandlung und-vernachlässigung, 17(2), 194-207.

Wilde, N. (2020). Mobbing - Ein Gruppenphänomen? In M. Böhmer \& G. Steffgen (Hrsg.), Mobbing an Schulen (S. 79-97). Springer VS.

Wolff, M. (2018). Schutz. In G. Graßhoff, A. Renker, \& W. Schröer (Hrsg.), Soziale Arbeit (S. 619-630). Springer VS.

Open Access Dieses Kapitel wird unter der Creative Commons Namensnennung 4.0 International Lizenz (http://creativecommons.org/licenses/by/4.0/deed.de) veröffentlicht, welche die Nutzung, Vervielfältigung, Bearbeitung, Verbreitung und Wiedergabe in jeglichem Medium und Format erlaubt, sofern Sie den/die ursprünglichen Autor(en) und die Quelle ordnungsgemäß nennen, einen Link zur Creative Commons Lizenz beifügen und angeben, ob Änderungen vorgenommen wurden.

Die in diesem Kapitel enthaltenen Bilder und sonstiges Drittmaterial unterliegen ebenfalls der genannten Creative Commons Lizenz, sofern sich aus der Abbildungslegende nichts anderes ergibt. Sofern das betreffende Material nicht unter der genannten Creative Commons Lizenz steht und die betreffende Handlung nicht nach gesetzlichen Vorschriften erlaubt ist, ist für die oben aufgeführten Weiterverwendungen des Materials die Einwilligung des jeweiligen Rechteinhabers einzuholen.

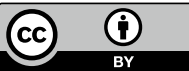

